\title{
Reviewers: 2013-2017
}

https://doi.org/10.1515/ael-2018-0005

The editorial board of CONVIVIUM wishes to thank all our reviewing scholars who contributed to our supportive peer-review process.

\section{List of Reviewers 2013-2014}

Angel, James

Ariff, Akmalia

Avi-Yonah, Reuven

Baker, Charles

Bavoso, Vincenzo

Beattie, Vivian

Broadbent, Jane

Burke, John JA

Buthe, Tim

Christensen, Mark

Corradi, Marco

Deluca, Anna

DeMott, Deborah

Duhamel, Jean-Christophe

Durtschi, Cindy

Dutta, Sahil

Dutta, Saurav

Fantacci, Luca

Ferri, Giovanni

Gabbioneta, Claudia

Guven-Uslu, Pinar

Hughes, Matthieu

Ikaheimo, Seppo

Jamal, Karim

Keay, Andrew

Krampf, Arie

Lyu, Kai

Marzo, Giuseppe

Masciandaro, Donato 
Mazzola, Pietro

Milot, Jean-Paul

Monsen, Norvald

Moore, Marc

Mortenson, Julian

Mussari, Riccardo

Mustonen, Esko

Ojo, Marianne

Ortiz, Horacio

Penman, Stephen

Pizzo, Michele

Polo Garrido, Fernando

Previts, Gary

Ramanna, Karthik

Rebérioux, Antoine

Richard, Chrystelle

Righi, Simone

Riva, Angelo

Robb, Alan

Schmidt, Reinhard

Sergakis, Konstantinos

Shaheen, Fadi

Stout, Lynn

Thoman, Lynda

Waibel, Michael

Weber, Olaf

Weinstein, Olivier

Williams, Cynthia

Williams, Paul

Woll, Cornelia

\section{List of Reviewers 2015-2017}

Ariff, Akmalia

Ashton, Philip

Avi-Yonah, Reuven

Azam, Rifat

Baker, Charles

Basu, Sudipta 
Bavoso, Vincenzo

Blad, Karin

Blair, Margaret

Bourghelle, David

Braun, Eduard

Butzbach, Olivier

Chabrak, Nihel

Chambost, Isabelle

Chan, James

Charron, Jacques-Olivier

Clarke, Thomas

Clausing, Kimberly

Corradi, Marco

Cullen, Jay

Edgley, Carla

Fantacci, Luca

Fligstein, Neil

Fukui, Yoshitaka

Gabbioneta, Claudia

Gallegati, Mauro

Garbarino, Carlo

Gialdini, Laurence

Gindis, David

Glover, Jonathan

Goldstein, Adam

Haberly, Daniel

Haslam, Colin

Herman, Barry

Hodges, Ron

Hommel, Michael

Hujii, Hideki

Ishikawa, Tomoko

Jamal, Karim

Jansson, Andreas

Kirman, Alan

Kleinman, Gary

Kuźniacki, Błażej

Lagneau-Monet, Paul

Lengnick, Matthias

Lienau, Odette 
Lin, Haijin

Mähönen, Jukka

Mallett, Jacky

Manes Rossi, Francesca

Marian, Omri

Mariotto, Carlotta

Mazzola, Pietro

Moe, Thorvald

Montagne, Sabine

Moretti, Delphine

Moutot, Philippe

Newberry, Susan

Nguyen, Lisa

Okata, Satoru

Oulasvirta, Lasse

Pelger, Christoph

Previts, Gary

Quaglia, Lucia

Quentin, David

Raffer, Kunibert

Ramanna, Karthik

Ricks, Morgan

Riva, Angelo

Rixen, Thomas

Robé, Jean-Philippe

Sabac, Florin

Schumacher, Julian

Seabrooke, Leonard

Sergakis, Konstantinos

Shaheen, Fadi

Sjåfjell, Beate

Soltes, Eugene

Stafford, Anne

Stevelman, Faith

Sucher, Sandra

Tang, Vicki Wei

Taylor, Mark

Thiemann, Matthias 
Torfason, Asgeir

Ugolini, Stefano

Waymire, Gregory

Weinstein, Olivier

Woll, Cornelia

Woschnack, Daniela

Yim, Andrew 